(C) 2021 IEEE. Personal use of this material is permitted. Permission from IEEE must be obtained for all other uses, in any current or future media, including reprinting/republishing this material for advertising or promotional purposes, creating new collective works, for resale or redistribution to servers or lists, or reuse of any copyrighted component of this work in other works.

This is not the version of record. The full version of Bulat, Pavel, Esakov, Igor, Grachev, Lev, Volkov, Konstantin and Volobuev, Igor (2021) Experimental study of air breakdown induced by subcritical streamer microwave discharge. IEEE Transactions on Plasma Science, 49(3), pp. 1041-1049 can be found at https://doi.org/10.1109/TPS.2021.3064286 


\title{
Experimental Study of Air Breakdown Induced by Subcritical Streamer Microwave Discharge
}

\author{
P.V. Bulat ${ }^{1,2}$, I.I. Esakov ${ }^{3}$, L.P. Grachev ${ }^{3}$, K.N. Volkov ${ }^{4, *}$, I.A. Volobuev ${ }^{1}$ \\ ${ }^{1}$ Baltic State Technical University, 190005, St Petersburg, Russia \\ ${ }^{2}$ Problemnaya laboratoriya "Turbomashiny", 198035, St Petersburg, Russia \\ ${ }^{3}$ Moscow Radiotechnical Institute of Russian Academy of Sciences, 117519, Moscow, Russia \\ ${ }^{4}$ Kingston University, SW15 3DW, London, United Kingdom
}

\section{Abstract}

Streamers are non-thermal filamentary plasmas developing in insulating mediums under the influence of strong external electric field. The subcritical streamer discharge is formed by a field with the intensity smaller than the minimum intensity leading to the air breakdown, and breakdown conditions are created by special initiators. Air breakdown in sub- and supersonic flows induced by the subcritical streamer discharge and the effect of the initiator shape on breakdown conditions are studied. The intensity of electric field of subcritical streamer induced breakdown has been shown to be smaller compared to that initiated by microwave discharge without initiator. The dependencies of critical intensity of the electric field on the pressure and location of initiator are reported. The air breakdown conditions are found at various dimensions of the metallic screen near which the initiator is installed.

\section{Keywords}

Air breakdown, microwave discharge, streamer discharge, plasma-assisted technology.

\section{Introduction}

Difficulties of mixing, ignition and combustion of hydrocarbon fuels in the high-speed flows are associated with relative large characteristic ignition and combustion times. A significant number of experimental, theoretical and computational studies investigating the application of plasma discharges as actuators for high-speed flow control have been performed for last years. The development of microwave electronics has led to the possibility of initiating an electrodeless microwave discharge in free space in a wide range of external conditions [1-4].

Various types of plasma discharges have shown promising characteristics in different applications including flow control in aerodynamics [5-7], ignition and combustion in propulsion engineering [8-13], stabilization of flames [14-17]. Characteristic propane ignition and combustion times in the supersonic flow with Mach number 2 could be decreased up to factor $2-5$ by plasma-assisted combustion $[8,9]$. Influence of the energy input of a microwave discharge on shock-wave structures and the flow around a body in a supersonic flow and the optimal discharge parameters to achieve the aerodynamic effect are studied in $[6,7]$. A model of an initiated microwave discharge in a linearly polarized wave beam is considered in [18]. It includes a complete set of kinetic reactions and takes into account heating and

\footnotetext{
${ }^{*}$ Corresponding author: k.volkov@kingston.ac.uk
} 
gas-dynamic rarefaction of the microwave channel. A detailed study of the plasma-chemical and gas-dynamic processes in the zone of the microwave discharge is performed in [19]. The fundamental phenomena of self-organization and filamentation of freely localized microwave discharges are investigated numerically in [20]. The study [21] is devoted to the breakdown of pulsed microwave discharges. Numerical calculation are carried out in [22] to investigate the space-time dynamics of the formation of a focused pulsed microwave discharge in air.

When a high-power electromagnetic pulse is focused at a point and interacts with a gas, the gas is heated to temperatures of thousands of degrees within several microseconds, breaking down and becoming highly ionized. This process, air breakdown, is accompanied by a light flash and generation of sound (acoustic shock wave).

There are various types of microwave discharges which have been realized depending on the pressure and electric intensity for different wavelengths [23-27]. Recent investigations have demonstrated a great potential of microwave discharges to ignite air/fuel mixtures at low-pressure $[12,13]$. In these applications, the discharges take the form of thin plasma filaments driven by highly non-linear ionizing waves (streamers). The limitations of source power supplies and pressure levels in plasma-assisted technologies technology make it necessary to use a subcritical streamer discharge. The main positive point of these studies is the high temperature in the discharge, which ranges from 1000 to $2000{ }^{\circ} \mathrm{C}$. Due to the use of the resonant method of amplifying the electric field, streamer discharges are ignited at the initial field strength which is much lower than the breakdown energy. Streamer discharges occur as a consequence of the high electric field induced by the fast variations of the net charge density ahead of an electron avalanche with large amplification.

Ignition of freely localized electrodeless microwave discharge at high gas pressure requires high levels of microwave energy in the electromagnetic beam. For example, at air pressure $p=$ 760 torr the breakdown takes place at $10^{6} \mathrm{~W} / \mathrm{cm}$. A local initiation of a discharge is provided by special facilities. Then, the streamer microwave discharge spreads in a considerable volume of the microwave beam. In this case the level of energy is less than the critical level of breakdown (subcritical microwave discharge).

Previous experiments have shown that the microwave discharge is of the streamer form at the high pressure in the subcritical $\left(E_{0} / E_{c}<1\right)$ and deep subcritical $\left(E_{0} / E_{c} \ll 1\right)$ fields. The temperature of plasma streamer channels that form the discharge has the scale no less than $2000 \mathrm{~K}$. In principle, the realization of such temperature is enough for the fuel ignition.

Numerical simulation of gas heating effects in a nanosecond-pulse streamer discharge interacting with a Mach 3 supersonic flat plate boundary layer flow is discussed in [28]. The plasma solver calculates all plasma dynamics, reactive chemistry and species transport effects. The gas pressure, temperature and velocity fields are calculated by the Navier-Stokes solver. Plasma and flow solvers are coupled at each time step resulting in a fully time-accurate simulation procedure. Rapid gas heating occurs in the electrode near-field region with gas temperature increase on the order of 100-200 K. The rapid heating of gas results in micro blast waves which propagate into the freestream and eventually decay. Plasma-assisted flame ignition in supersonic flow over a flat plate are discussed in [29].

Computations of air glow surface plasma in the presence of supersonic flow are presented in [30]. The glow discharge model is based on a self-consistent, multi-species, continuum description of the plasma with finite-rate chemistry effects. This model is coupled with a compressible Navier-Stokes solver. The effect of the plasma discharge on Mach 3 supersonic flow at freestream pressure of 18 torr and the corresponding effects of the flow on the discharge structure is studied by examining the species concentrations and the gas temperature. For the case studied, the primary effect of the plasma on the supersonic flow is volumetric heating. Different ion species are found to be dominant in the absence and presence of super- 
sonic flow, highlighting the importance of including finite-rate chemistry effects in discharge models for understanding plasma actuator physical phenomena.

Multi-scale streamer discharge simulation is performed in [31]. The numerical strategy is applied to simulation of propagation of streamer discharges and nanosecond repetitively pulsed discharges. A deep sub-critical streamer discharge is used in [32] (wavelength is 12.4 $\mathrm{cm})$ to ignite air/propane mixture in the supersonic flow at high altitudes.

Creation of longitudinal plasma formation in cold supersonic air flow is difficult due to fast plasma cooling by air stream and discharge plasma instabilities. Experimental data on the ignition of a pulsed triggered microwave streamer discharge in a supersonic air jet are presented in [23]. A microwave streamer discharge at wavelength of $8.5 \mathrm{~cm}$ is produced in air with pressure of 60 torr, and a triggered variant of discharge with a developed structure is produced with electric field strength of $1.5 \mathrm{kV} / \mathrm{cm}$ and duration of pulse larger than 10 $\mu \mathrm{s}$. The resonant portions of the streamers which appear in the discharge front radiate intensely in the visible spectral range for a time of several microseconds. These experiments on the ignition of microwave streamer discharges in supersonic gas jets with velocities of up to $500 \mathrm{~m} / \mathrm{s}$ have shown that discharges under these conditions are still streamer discharges. Some semi-empirical correlations describing various forms of streamer discharge are proposed in [24], and experimental results are presented in [25-27].

Processes that control air breakdown remain unresolved and introduce significant uncertainties into modelling and simulation. The most important parameter for practical applications is the threshold intensity of microwave radiation required for air breakdown. The study focuses on investigation of possibilities of the use of microwave radiation to initiate breakdown in air. The results of experimental studies related to air breakdown induced by microwave radiation are presented. To initiate the air breakdown, the subcritical streamer discharge is used. The discharge is formed by a field with the intensity smaller than the minimum pulse intensity leading to the air breakdown.

Previous studies have been focused on air breakdown by the initiators located near the dielectric screen. The submitted paper deals with the air breakdown induced by streamer discharge with the use of initiator located near the metallic screen. To our best knowledge, this research is not part of the papers published in the open literature. Another important issue from both theoretical and practical points of view is the minimal area of the screen which is sufficient to induce air breakdown. This study is also a part of the presented paper. Experimental data included in the paper is obtained at different lengths and widths of the screen, and breakdown conditions are determined.

\section{$2 \quad$ Experimental setup}

The experiments are carried out using the test rig designed at the Moscow Radio-Technical Institute of Russian Academy of Science (MRTI RAS). The test rig is similar to that used and described in [32]. The test rig includes the vacuum part, aerodynamic part forming lowor high-speed flow, microwave part forming electromagnetic beam, elements of discharge initiation and measuring equipment.

The test rig presented in the Figure 1 shows the flow channel with a high-speed air valve at the inlet, microwave generator waveguide, pressure and temperature measurement system and exhaust system placed in a vacuum chamber [32]. A manifold for propane injection to the flow channel is placed before air valve. The mass flow rate is controlled by fuel valve (it is not used in the current study) and pressure in the tank. The experimental setup is equipped with Phantom v.251 high-speed camera (a speed is 510000 frames/s), a number 
of fast-response pressure transducers, photo-sensors and current voltage sensors. The measuring equipment includes manovacuummeters to measure static pressure in the working chamber with accuracy \pm 1.5 torr, high-speed electronic sensors to measure pressure variation in the working chamber, Pitot tube with corresponding electronic scheme to measure stagnation pressure and thermocouples to measure flow temperature. The thermocouple and the pressure sensor signals are recorded with Instek GDS-830 oscilloscope (a typical speed up is 105 torr/s and accuracy is \pm 1.5 torr).

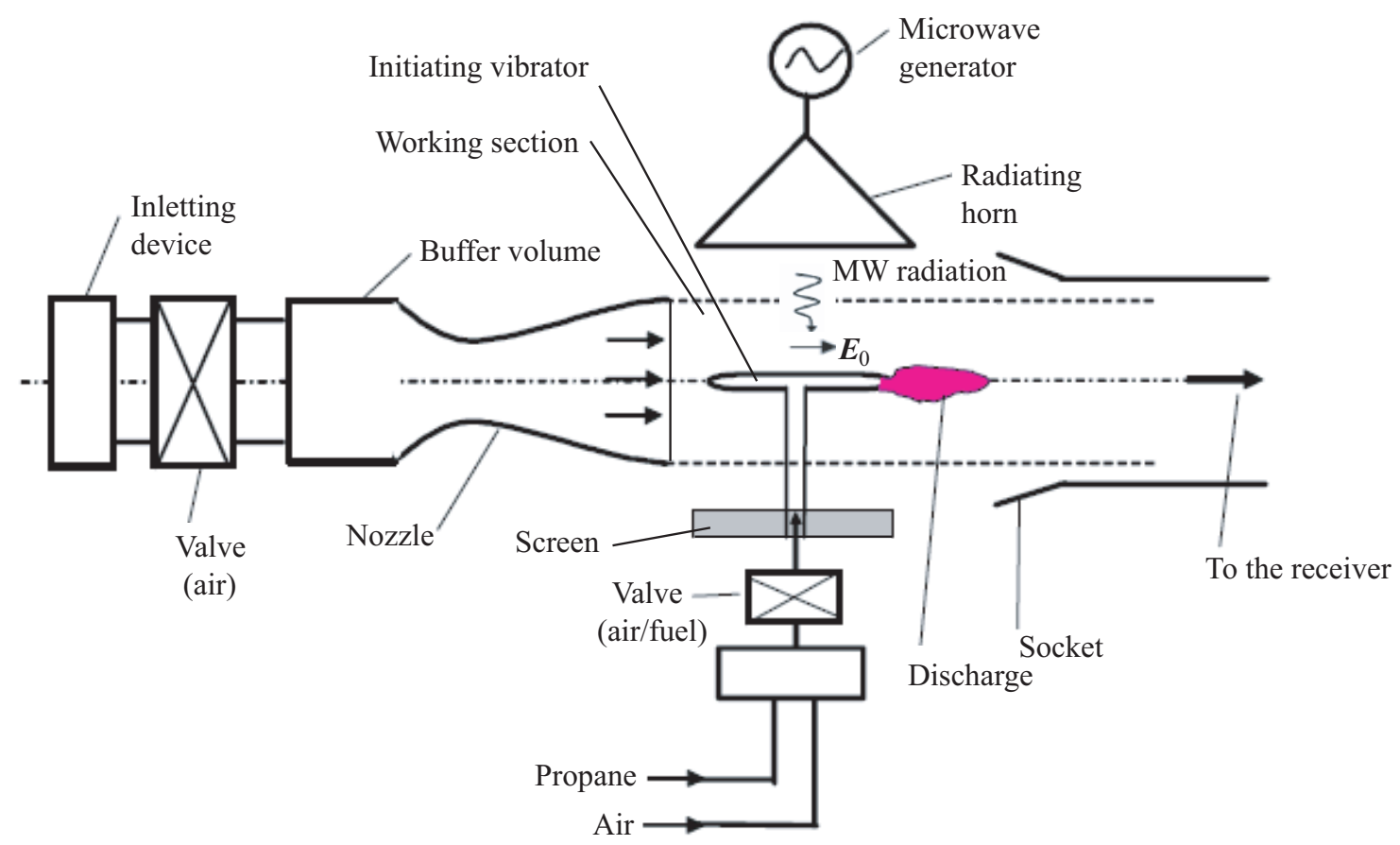

Figure 1. Test rig

The experimental tests are performed in a pulsed regime. Electromechanical valve is located at nozzle inlet. The triggering signal starts the oscilloscope measurement, and air valve opens after $0.2 \mathrm{~s}$. The microwave discharge is initiated after $0.1 \mathrm{~s}$ and burns $0.4 \mathrm{~s}$.

Vacuum part of the test rig consists of the working chamber and large hermetic tankreceiver. The working chamber is a horizontally located metal cylinder with flat edges and window on its side surface. The diameter of the working chamber is $0.7 \mathrm{~m}$, and its length is $1 \mathrm{~m}$. The working chamber has a volume of $0.4 \mathrm{~m}^{3}$. The receiver has a rectangular shape with dimensions of $1.25 \times 1.25 \times 2.5 \mathrm{~m}^{3}$ (its volume is $4 \mathrm{~m}^{3}$ ). There is a spherical transitional section between the working chamber and the receiver with a volume of $0.15 \mathrm{~m}^{3}$. The entire volume of the vacuum part is $4.2 \mathrm{~m}^{3}$. Pressure varies from 10 to 760 torr.

The high-speed flow is formed by the axisymmetric Laval nozzle. The nozzle throat is $23 \mathrm{~mm}$. The inlet diameter of the nozzle is $96 \mathrm{~mm}$, and the length of its converging part is $100 \mathrm{~mm}$. The outlet diameter of the nozzle is $30 \mathrm{~mm}$, and the length of its diverging part is $80 \mathrm{~mm}$.

The air speed at the nozzle outlet varies from 10 to $500 \mathrm{~m} / \mathrm{s}$. The flow is imposed by the pressure difference between inlet section of the nozzle and vacuum chamber. The total pressure at the inlet section of the nozzle is fixed at 750 torr. The total temperature is $300 \mathrm{~K}$. The static pressure in the working chamber varies from 30 to 150 torr. Supersonic jet is formed if a static pressure in the working chamber is less than 380 torr. For typical experimental conditions, the Mach number is 2 and flow speed is $500 \mathrm{~m} / \mathrm{s}$, static pressure in the working chamber is 97 torr, air temperature is $160 \mathrm{~K}$, the concentration of molecules is 
$6 \times 10^{18} \mathrm{~cm}^{3}$. The supersonic flow has a core with typical of $1.8 \mathrm{~cm}$ at a distance of $12 \mathrm{~cm}$ from the nozzle exit. The pressure in the working chamber is maintained constant during 1 $\mathrm{s}$, and then it starts to grow.

The working chamber and the receiver are connected by conic-cylindrical section. This section moves along the centerline of the chamber. It is mounted at different distances from the nozzle exit varying from 0.05 to $0.5 \mathrm{~m}$. The internal diameter of the cylindrical section is $0.07 \mathrm{~m}$, and its length is $0.7 \mathrm{~m}$. The conical section is $0.22 \mathrm{~m}$ long with a maximum inlet diameter $0.14 \mathrm{~m}$. The conic-cylindrical section is installed and configured in a way to prevent the backward air flow from the receiver to the working chamber. The parameters of the jet are kept constant for a time of about $2 \mathrm{~s}$.

Electromagnetic beam is generated with a magnetron. Its output power is $1.5 \mathrm{~kW}$, and wavelength is $\lambda=12.3 \mathrm{~cm}$ (this wavelength is one of the most common for microwave generating devices [27]). A typical outlet area of magnetron is $9 \times 9 \mathrm{~cm}^{2}$. Linearly polarized electromagnetic beam formed in the working chamber is normal to flow direction. The vector of electrical component of electromagnetic field $\boldsymbol{E}_{0}$ is parallel to flow velocity and has a magnitude of $E_{0}<7.2 \mathrm{kV} / \mathrm{cm}$ at the focus. The transverse distribution of the intensity of electromagnetic radiation of the beam is close to Gaussian with a characteristic size of 5 $\mathrm{cm}$. It is uniform along the beam centerline over a length of a few centimeters. The pulse duration is $40 \mu \mathrm{s}$.

The discharge ignition occurs in the working section of the channel. Vibrator is a copper pipe with the length $2 L$ and flat faces. The outer diameter of the vibrator is $2 a$ which equals to $4,6,10,12$ and $18 \mathrm{~mm}$ in the experiments. The vibrator is placed horizontally (its axis is aligned with the direction of air flow) and symmetrically with respect to microwave beam. The vibrator is mounted on a pylon that is $2 \mathrm{~mm}$ thick and $10 \mathrm{~mm}$ wide. The total obstruction of the pass is $14.7 \%$. The pylon is located on a flat conducting screen. The distance from the vibrator axis to the screen is $H$.

\section{Discharge initiation}

Characteristic feature of the streamer discharge is the formation of a spatially spreading structure [17]. Streamer discharge has a complicated composition of thin plasma filaments in the integrated photographs with exposure times which is larger than duration of microwave pulse (Figure 2). Plasma filaments that make up the discharge are continually lengthening and branching. Plasma filaments propagate toward the microwave source at the speed of several kilometers per second, and the distance between each filaments is about one-fifth of the wavelength of the incident microwave radiation.

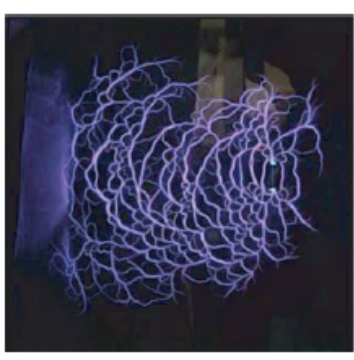

a)

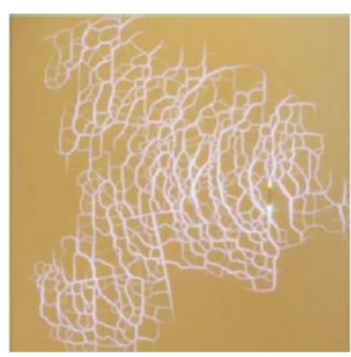

b)

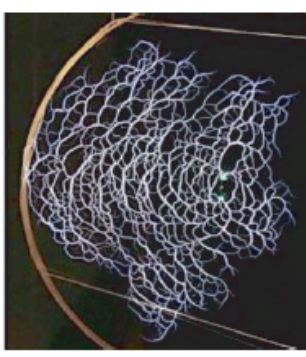

c)

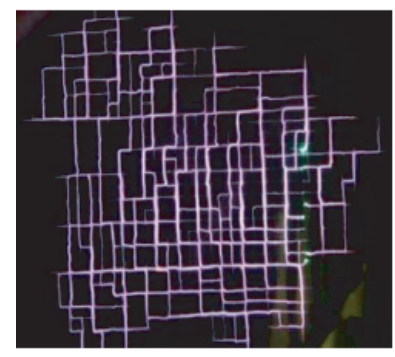

d)

Figure 2. A streamer discharge in a free space (a), on a laminate surface (b), on a glass surface (c) and on a capron net (d)

With external similarity to the diffuse discharge, the attached deep subcritical streamer 
discharge is effectively heated by microwave radiation, while the diffuse discharge does not absorb microwave radiation and remains cold. Under resonance conditions, a large induced current arises in the conductor, and gas ionization occurs in the vicinity of the initiator, and plasma channels are formed, which strongly heat the surrounding gas. If the overheated channel cannot escape beyond the diffuse cloud, then the discharge remains attached. With a sufficiently high intensity of the electric field, a spatial tree-like structure develops from the overheated channel.

Streamer discharges induced by quasi-optical microwave beams in air are classified in [1] by analyzing a series of time-integrated frames (the exposition time exceeds the time of pulse) operating at different initial field strengths and air pressures. Diffuse, streamer, overcritical, subcritical and deep subcritical streamer discharges are distinguished. The domains of existence of each form of discharge are defined as functions of air pressure and electrical field strength ratio $E_{0} / E_{c}$ (wavelength is fixed), where $E_{0}$ is the electric field of the microwave beam in the discharge region and $E_{c}$ is the critical breakdown of the electric field. It is possible to ignite a self-sustained discharge without any initiated settings if $E_{0}>E_{c}$, while at $E_{0}<E_{c}$ a discharge has to be initiated with some additional settings such as a special designed metal vibrator or a preliminary discharge introduced by laser. The difference between the subcritical and deep subcritical discharges is that the streamers remain attached to the initiator for deep subcritical discharge. The diffuse discharge plasma in the subcritical region also remains attached to the initiator.

The experimental procedure is as follows. A relatively high gas pressure $p_{c}$ is established in the working chamber, at which microwave breakdown is not observed. Then, pressure decreases with a step of 30 torr until the breakdown is recorded. After that pressure increases by 30 torr, a time delay of five minutes is made and the pressure decreases to the breakdown value $p_{c}=p_{b}$ with a step of 3 torr. To ignite the discharge, an aluminium cylindrical vibrator located at the distance $H=120 \mathrm{~mm}$ from the screen is used (Figure 3). The length and diameter of the vibrator are $2 L$ and $2 a$. The length of vibrator does not exceed $5 \mathrm{~cm}$ (it is smaller than the diameter of electromagnetic beam). Cylindrical vibrator with spherically rounded edges (Figure 3b) and cylindrical vibrator with one spherically rounded edge and with another cone edge (Figure 3c) are also applied to initiate air breakdown.

The minimum amplitude of the initial field at which vibrator still initiates air breakdown, $E_{0}=E_{b}$, is determined. The control measurements show that the amplitude of electric field is $E_{0}=50 \mathrm{~V} / \mathrm{cm}$ at the distance $H_{0}=77 \mathrm{~mm}$ from the source of electromagnetic radiation. The metallic screen increases this value up to $E_{0}=100 \mathrm{~V} / \mathrm{cm}$. Time of pulse is $0.2 \mathrm{~s}$ in both cases.

\section{Structure of streamer discharge}

Air flow is created in the test section of the experimental setup, and sub-critical streamer discharge is ignited by the initiator. Streamer speeds from experimental observations (symbols) and calculations based on semi-empirical correlations from [24] (solid lines) are presented in the Figure 4 (in the region of initiator).

The structure of triggered subcritical microwave streamer discharges observed in the experiments and the influence of supersonic air flow on a shape of subcritical microwave discharge is shown in the Figure 5. The radiation propagates from right to left and vector of electrical field lies in the plane of the figure. The length of the initiator is a scale length for the image. Air pressure is 100 torr and electric field strength is $1.7 \mathrm{kV} / \mathrm{m}$. The trigger is clearly visible in these pictures. Vaporization takes place at the point where surface of initiator 


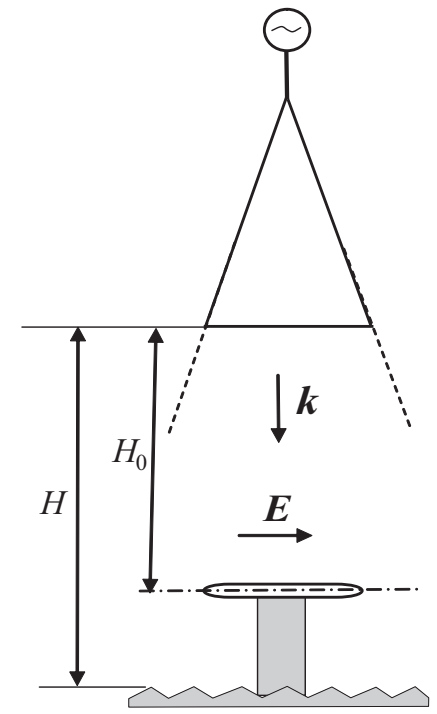

b)

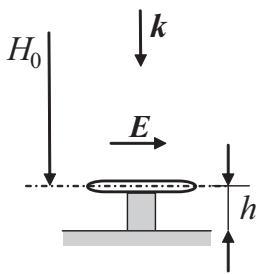

c)

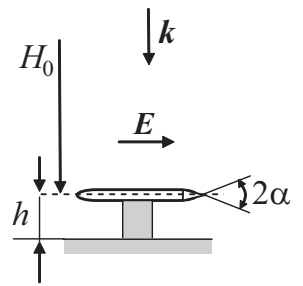

a)

Figure 3. Geometry of cylindrical vibrator (a), vibrator with spherically rounded edges (b) and vibrator with one spherically rounded edge and with another cone edge $(\mathrm{c})$
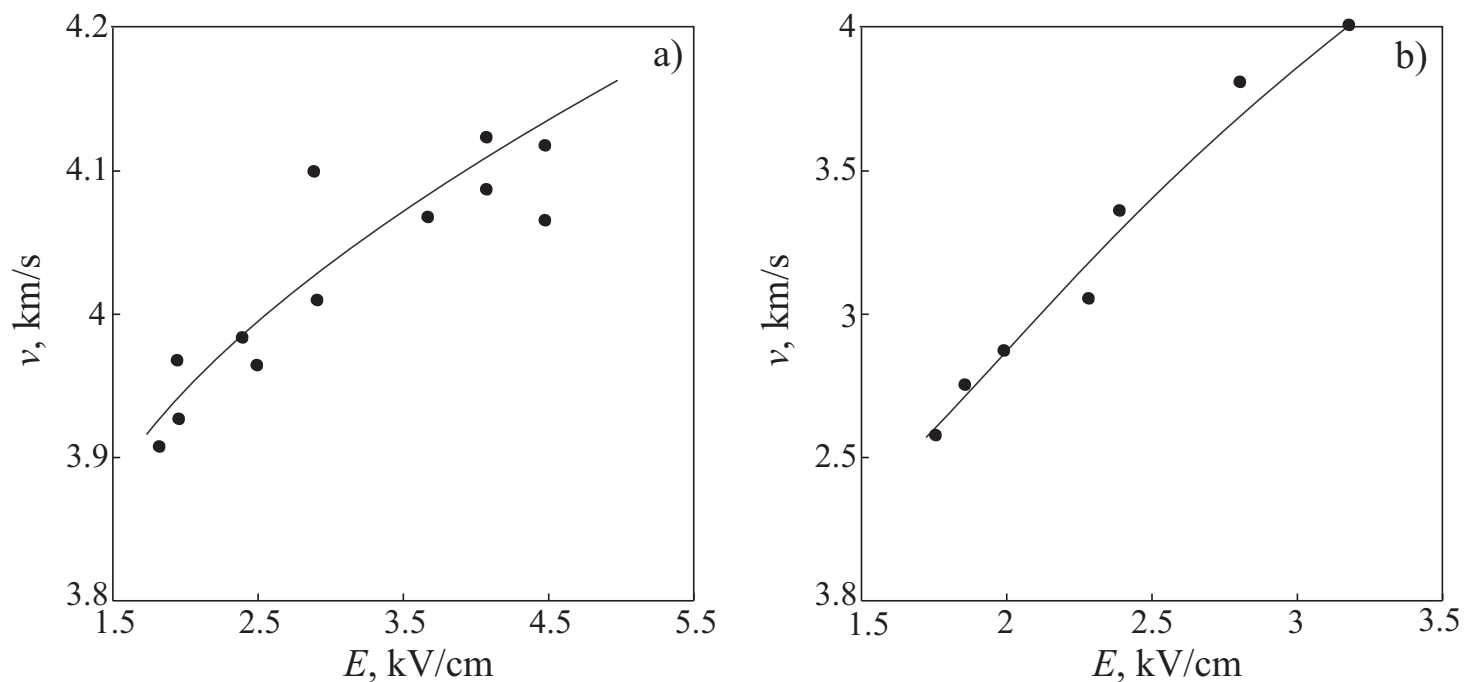

Figure 4. Dependance of the propagation speed of streamer discharge on electric field strength in a quiescent air (a) and in a quartz tube (b) at atmospheric pressure

comes into contact with the high temperature plasma channels (Figure 5a). The streamer discharge in the supersonic flow does not change its shape, but its plasma channels are blurred (Figure 5b). In the supersonic flow, the discharge stretches out significantly along the flow and occupies a larger volume. The lower end of the dipole is subjected to vaporization, and the flow has not reduced the temperature of the plasma channels significantly.

Discharge has a complicated filamentary structure. The discharge is shaped as a net of very thin luminous filaments with bright fragments, which length approximately equals half wave length of radiation. The discharge consists of thin filaments self-organized into electrodynamic resonant vibrators and loops, rising one from another and propagating away from initiating point. The observations show that filament fragments of discharge net do not exist simultaneously but arise one from another. Each filament fragment at end stage of its evolution generates the cylindrical shock wave with initial Mach number equaling 1.5-2. 


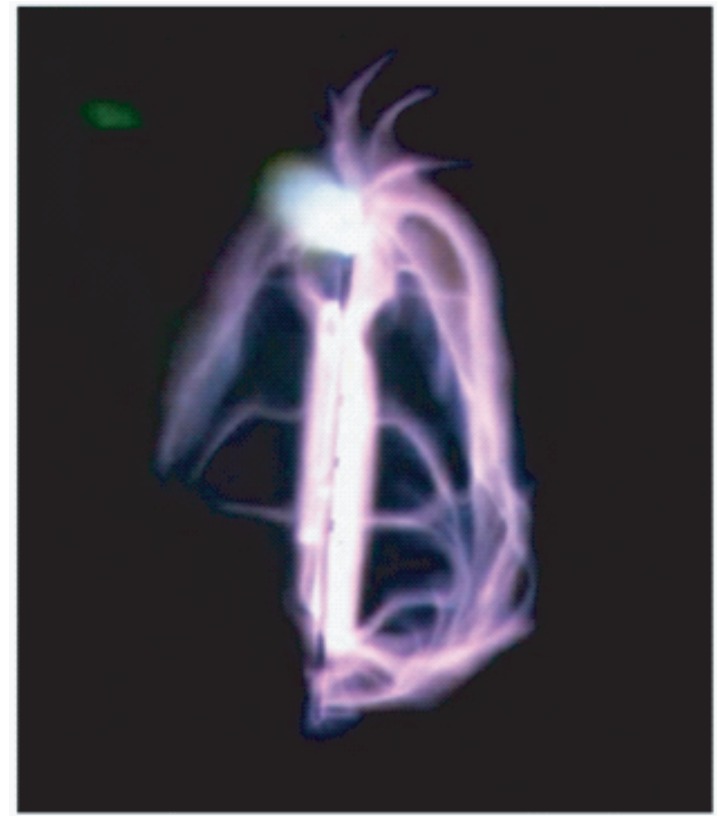

a)

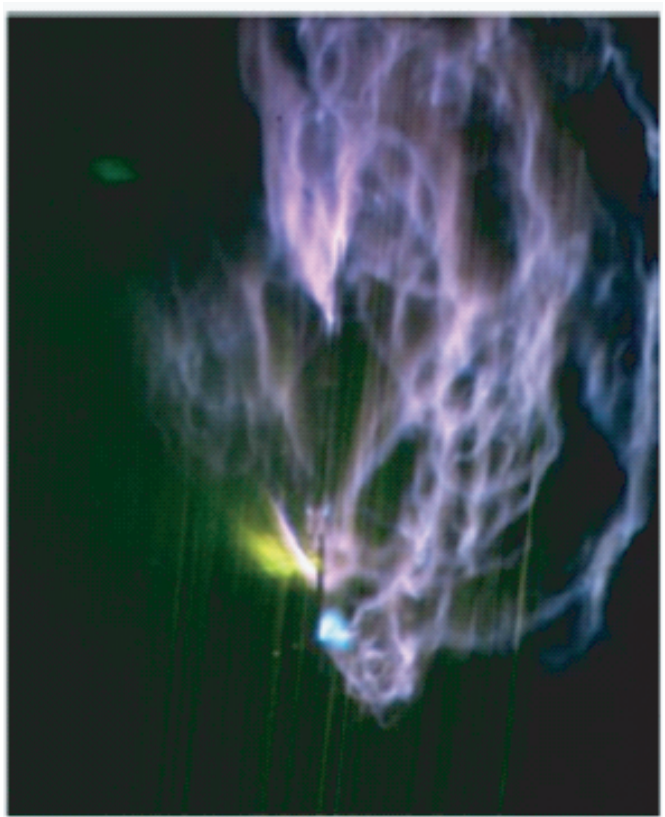

b)

Figure 5. Subcritical streamer discharges attached to initiator in a quiescent air (a) and supersonic flow at the velocity $500 \mathrm{~m} / \mathrm{s}$ (b)

It means that gas temperature in filaments is high. The material of surface at poles of the vibrator evaporates, and temperature of the streamer channels exceeds $2000 \mathrm{~K}$. The 5-10 $\mathrm{cm}$ wavelength radiation and $0.3-1$ atm air pressure are good conditions for observation of the discharge. It is discovered experimentally that after creation the raised discharge is supported by radiation with electric field amplitude much less than critical value.

Figure 5a shows there is the streamer channel that creates the loop connecting the vibrator ends. The discharge presented in the Figure 5b is qualitatively different. Deep subcritical discharge in the flow has a form of the system of luminescent channels stretched along the flow. The character of changing of the structure of this discharge has the qualitative explanation. The streamer channels in the air flow are lubricated along it, whereas at zero air velocity they have clear outlines.

A streamer discharge with a developed spatial structure both in free space and on the surface of the screen (Figure 6) is not blown away by the flow at a Mach number of up to 2, but only slightly diffuses $(E=3.7 \mathrm{kV} / \mathrm{cm})$.

The light near ends of the initiator has a color that differs from the light of the streamers. It is light of the evaporated metal. It means that temperature of discharge at that place is more than temperature of the metal evaporating at appropriate pressure. In the conditions of the experiment the vaporization of the surface on the initiator's poles begins at $p>250$ Torr. It starts from this pressure when the gas temperature inside the streamer plasma channels has the value more than the evaporation temperature (about $2500 \mathrm{~K}$ ) and energy coming to the vibrator's surface is enough for its vaporization.

In free space, the vibrator breaks down air at a pressure of about 370-380 torr. When the vibrator is placed above the metal screen in the antinode of the field at a distance of $h=\lambda / 4$ from the screen, the breakdown occurs at atmospheric pressure. With the approach of the vibrator to the metal screen at a distance less than $h \ll \lambda / 4$, the amplification coefficient of the electric field increases in comparison with the vibrator in free space. In this case, the resonant length of the vibrator increases.

When the vibrator is placed directly on the surface of a dielectric plexiglass screen with 


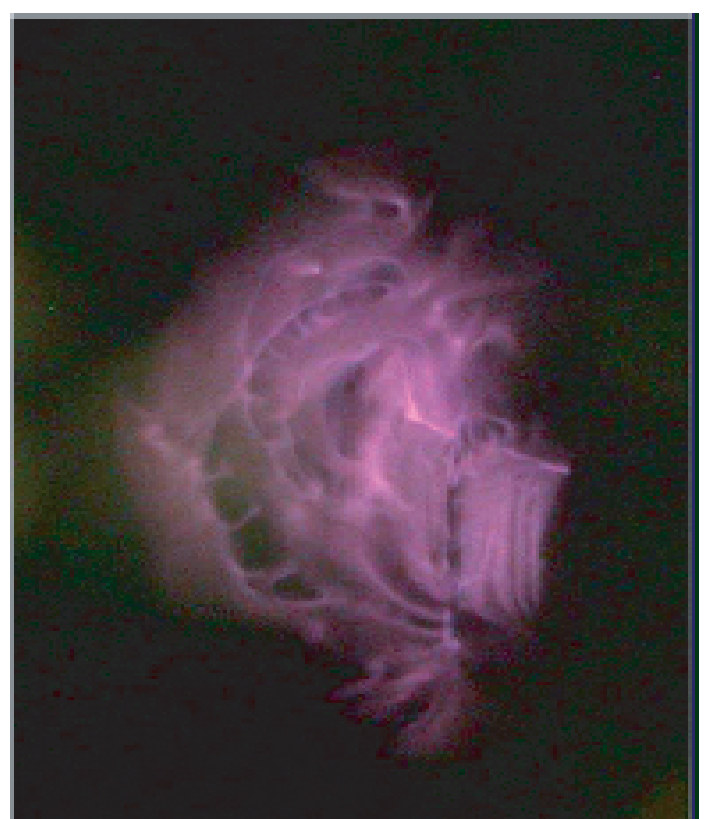

a)

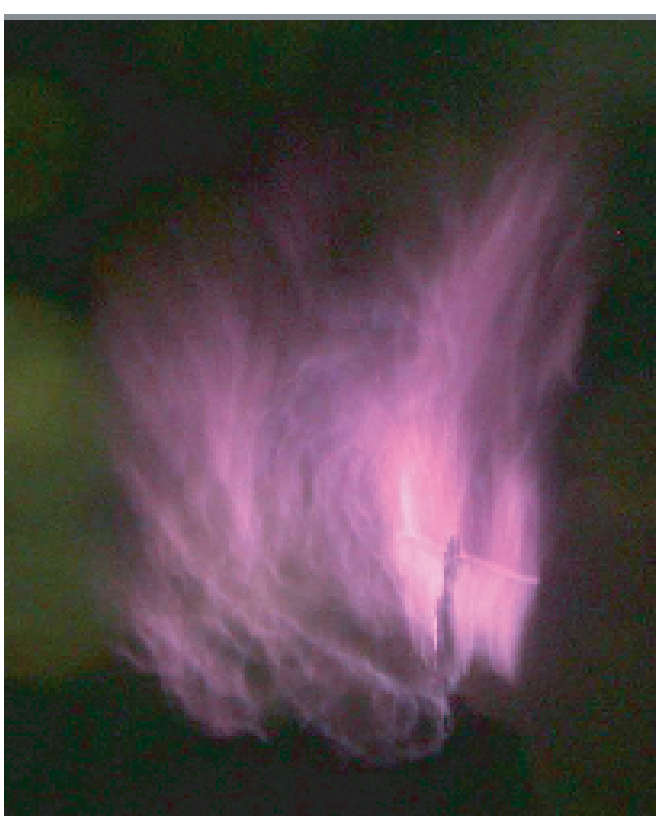

b)

Figure 6. Subcritical streamer discharges on a surface of dielectric screen in a quiescent air (a) and supersonic flow at the velocity $500 \mathrm{~m} / \mathrm{s} \mathrm{(b)}$

a thickness of $4.25 \mathrm{~mm}$, the field in the gap between the end of the resonator and the screen is more than two times higher than at the ends of the vibrator placed near or far from the screen. The gradual removal of the vibrator from the screen leads to a decrease in the field in the gap between it and the screen. A decrease in the distance between the dielectric screen and the vibrator leads to a decrease in the resonance length, and not to its increase, as is the case with a metal screen. So, the resonant length in free space is about $54.2 \mathrm{~mm}$, while on a plexiglass screen it is only $45.2 \mathrm{~mm}$, and on a ceramic screen is $34.5 \mathrm{~mm}$.

The photographs of discharges over an aluminum screen at different flow rates are presented in Figure 7. The discharge area is covered from above with a transparent plexiglass sheet. The order of the discharge length is about $1 \mathrm{~cm}$ at a speed of $1 \mathrm{~m} / \mathrm{s}$ and about $3 \mathrm{~cm}$ at $500 \mathrm{~m} / \mathrm{s}$. The discharge temperature is over $3000 \mathrm{~K}$. The power absorbed by the discharge increases with increasing speed and, accordingly, the size of the discharges, in the range from about 15 to $60 \mathrm{~W}$.

a)

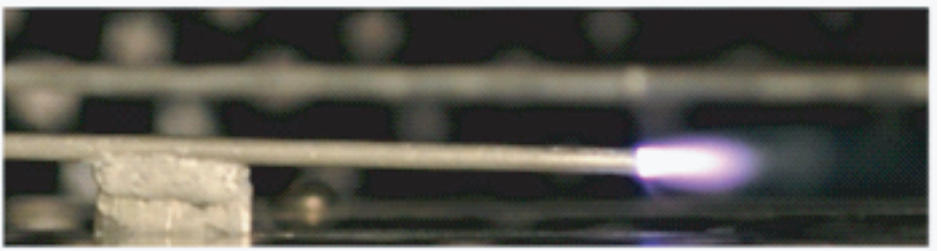

b)

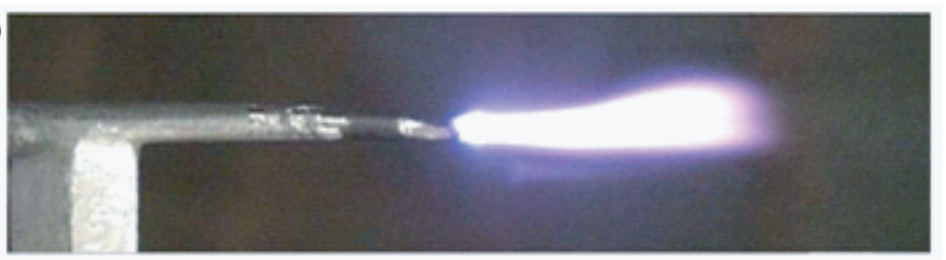

Figure 7. Subcritical streamer discharges in air flow at the velocity $1 \mathrm{~m} / \mathrm{s}$ (a) and $500 \mathrm{~m} / \mathrm{s}(\mathrm{b})$. The initiator is placed on a distance $h=\lambda / 4$ from the aluminium screen 


\section{$5 \quad$ Results and discussion}

Experimental dependencies of the critical field strength on vibrator length are presented in the Figure 8 for aluminium cylindrical vibrator with spherically rounded ends. The measurements are carried out at a constant air pressure in the chamber of $1 \mathrm{~atm}$. The minimum critical field strength increases with decreasing vibrator diameter, and position of the minimum approaches to $\lambda / 2$. For example, the resonant length of the vibrator $2 L_{r}$ with $2 a=2.5 \mathrm{~mm}$ is approximately $36 \mathrm{~mm}$, which corresponds to the ratio $2 L_{r} /(\lambda / 2)=0.8$. In this case, a vibrator initiates breakdown of atmospheric air at $E_{0}=250 \mathrm{~V} / \mathrm{cm}\left(E_{c} / E_{0}=130\right)$.

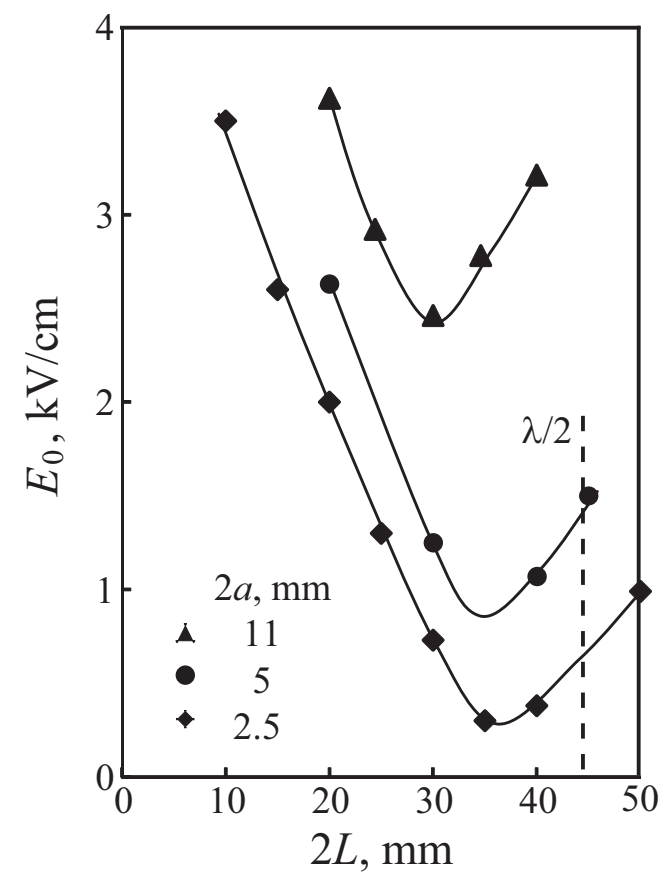

Figure 8. Dependence of the critical field strength on the vibrator length

The experimental results showing pressure effect on air breakdown are presented for cylindrical vibrator with spherically rounded edges in a free space (Figure 9), cylindrical vibrator with spherically rounded edges mounted at the distance $h=\lambda / 4$ from the screen (Figure 10), cylindrical vibrator with one spherically rounded edge and with another cone edge mounted at the distance $h=\lambda / 4$ from the screen (Figure 11). Symbols correspond to experimental data, and solid lines correspond to the best fitting of the experimental measurements. In these experiments, data points for breakdown pressure significantly deviate from the fitting line. This is because it is difficult to maintain the position of the vibrator and geometrical parameters of the vibrator-exciting beam throughout many successive experiments.

The results presented in Figure 10 show that the vibrator breaks down air at pressure $p=3.6 \times 10^{4} \mathrm{~Pa}$ (line 3 ). In this case it is possible to estimate its corresponding field at the pole of the vibrator as $E=42 p[\mathrm{~V} / \mathrm{cm}]$. Therefore, $E=2 \times 10^{4} \mathrm{~V} / \mathrm{cm}$ and $E / E_{0}=148$ for the measured value of $E_{0}$. The results corresponding to the line 1 show that in the range $2 L=47.5-48.5 \mathrm{~mm}$, the vibrator, initiates air breakdown under atmospheric pressure. The resonance length of the vibrator is $48 \mathrm{~mm}$ in this case. This length is shorter than the length of the vibrator placed in a travelling microwave.

At a relatively large sharpening angle of $2 \alpha=80^{\circ}$ of cone vibrator of a resonant length, air breakdown takes place at air pressure of 360 torr, which is 3 times larger than that for 


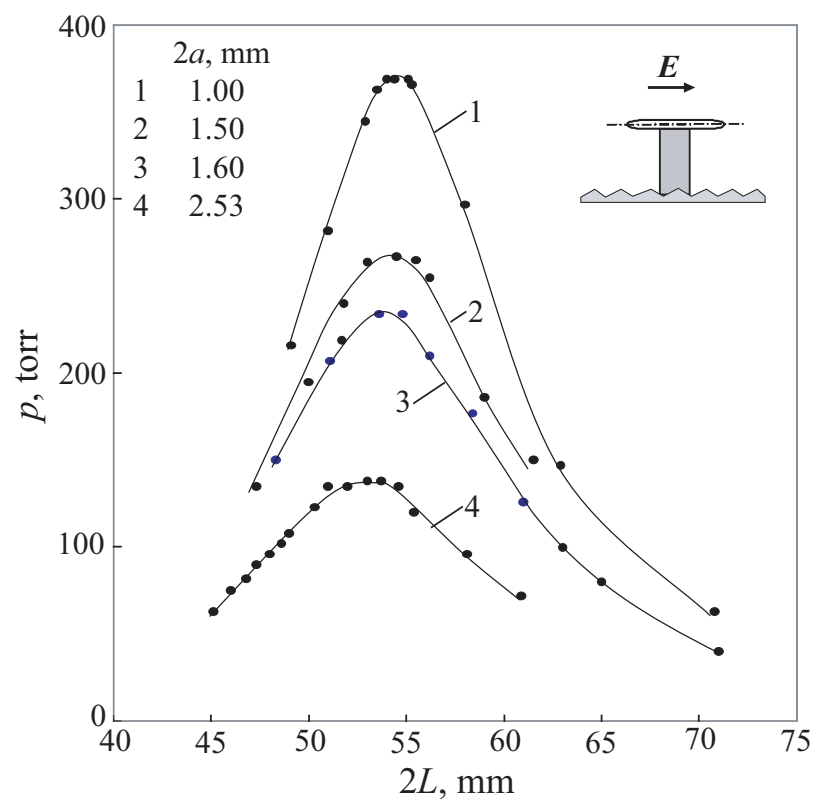

Figure 9. Dependance of air breakdown pressure on vibrator length for cylindrical vibrator with spherically rounded edges

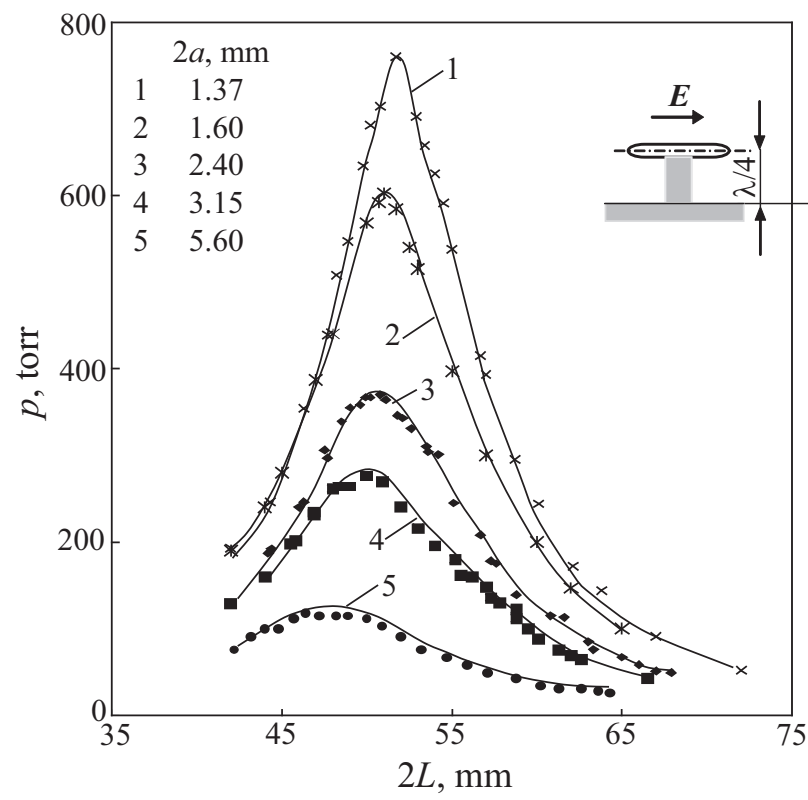

Figure 10. Dependance of air breakdown pressure on vibrator length for cylindrical vibrator with spherically rounded edges mounted at the distance $h=\lambda / 4$ from the screen

a vibrator with spherically rounded edges. The ability of cone vibrators with smaller angles to initiate air breakdown increases inversely proportional to the cone angle.

It is possible to initiate air breakdown at the level of the initial field strength in the electromagnetic beam $E_{0}$, which is significantly lower than the critical level, $E_{c}$ (in a deep subcritical field) by bringing the vibrator closer to the screen at distance $h<\lambda / 4$. The experimental results are shown in the Figure 12. Diameter of the vibrator is $2 a=3 \mathrm{~mm}$. Vibrator and screen are made from aluminium. The electric field strength is $E_{0}=50 \mathrm{~V} / \mathrm{cm}$. The length of the vibrator varies at each distance from the axis of the vibrator to the screen, $h$. For $h>10 \mathrm{~mm}$, the breakdown region is localized at the edges of the vibrator almost 


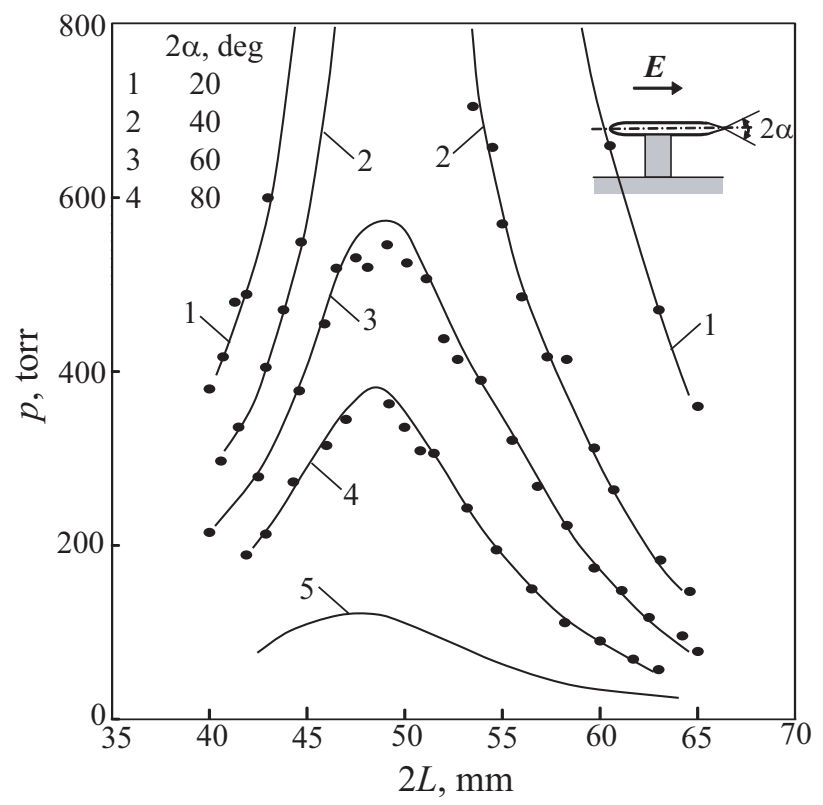

Figure 11. Dependance of air breakdown pressure on vibrator length for cylindrical vibrator with one spherically rounded edge and with another cone edge mounted at the distance $h=\lambda / 4$ from the screen $(2 a=5.6 \mathrm{~mm})$. Line 5 corresponds to cylindrical vibrator with spherically rounded edges in a free space

symmetrically about its axis, and for $h>10 \mathrm{~mm}$, they are localized already between the polar surfaces of the vibrator and the screen surface. There is an optimal size at which the ratio $E_{p} / E_{0}$ is maximum. The measurements gives optimal value for $h=6 \mathrm{~mm}$.

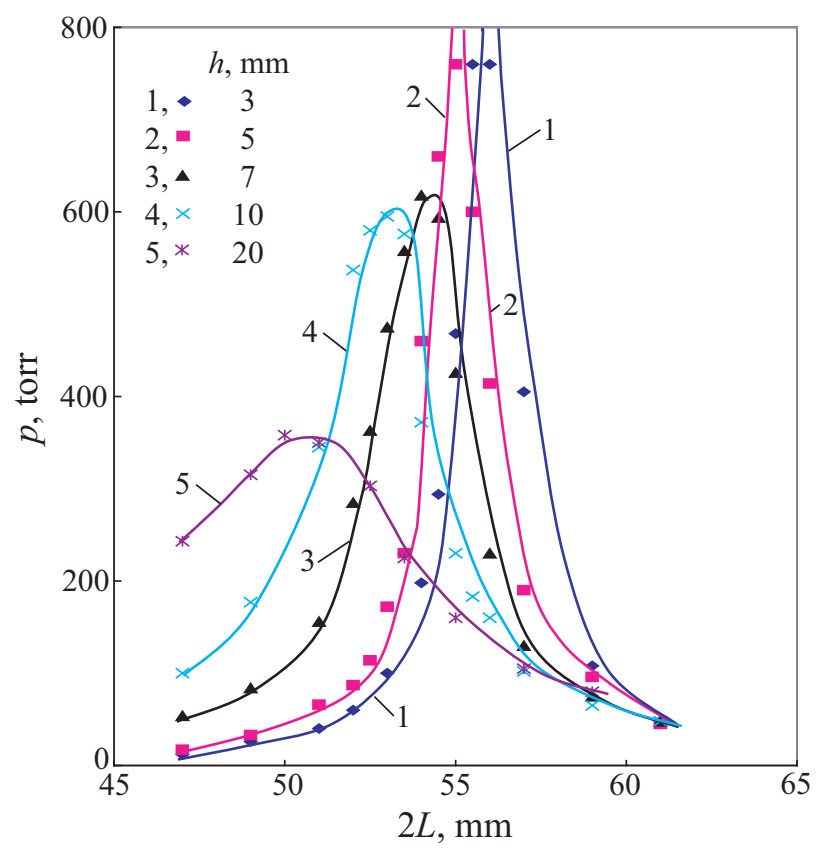

Figure 12. Dependance of air breakdown pressure on vibrator length for cylindrical vibrator with spherically rounded edges mounted at the various distances from the screen

Experiments with varying the electric field strength and air pressure show that the ability of electromagnetic vibrators to initiate air breakdown has a resonant character. The 
critical field amplitude at presence of an initiator is proportional to gas pressure at relatively high pressure of air.

Placing a small electromagnetic vibrator in a microwave beam allows to initiate air breakdown with a field strength in the microwave beam, $E_{b}$, which is significantly smaller than the critical breakdown strength, $E_{c}$. The electric field strength at the spherically rounded ends of the vibrator, $E_{p}$, is ten times larger than the initial field strength, $E_{0}$, if the length of the vibrator $2 L$ is four times its diameter $2 a$. Increasing the length of the vibrator in comparison to the wavelength of electromagnetic radiation, $\lambda / 2$, leads to resonant raising the ratio $\chi=E_{p} / E_{0}$, and air breakdown is induced with a significantly larger $\chi$. The amplification of the field by a vibrator placed on a dielectric screen is approximately equivalent to a vibrator fixed above a metal screen at a distance $h=\lambda / 4$.

The minimal dimensions or area of the screen $b \times c$ ensuring streamer induced air breakdown is of a great practical importance. The initiator of the resonance length $l=56$ $\mathrm{mm}$ is located near the screen at $h=5 \mathrm{~mm}$ from it. Diameter of the metallic vibrator is $d=2.25 \mathrm{~mm}$. Experiments are carried out at various lengths $b$ and widths $c$ of the screen. Decreasing $b$ or $c$ of the screen is made at the fixed another dimension which equals $100 \mathrm{~mm}$. The distance from the screen to the source of the microwave radiation is $H=125 \mathrm{~mm}$.

The dependence of the field amplification coefficient at the vibrator edge on the screen dimensions is presented in Figure 13 at different lengths and widths of the screen. The results obtained show that the breakdown pressure starts to decrease at $b<62 \mathrm{~mm}$ at the fixed $c=100 \mathrm{~mm}$. The symbols $\square$ show how the field amplification factor changes at various $c$ and the fixed $b=62 \mathrm{~mm}$.

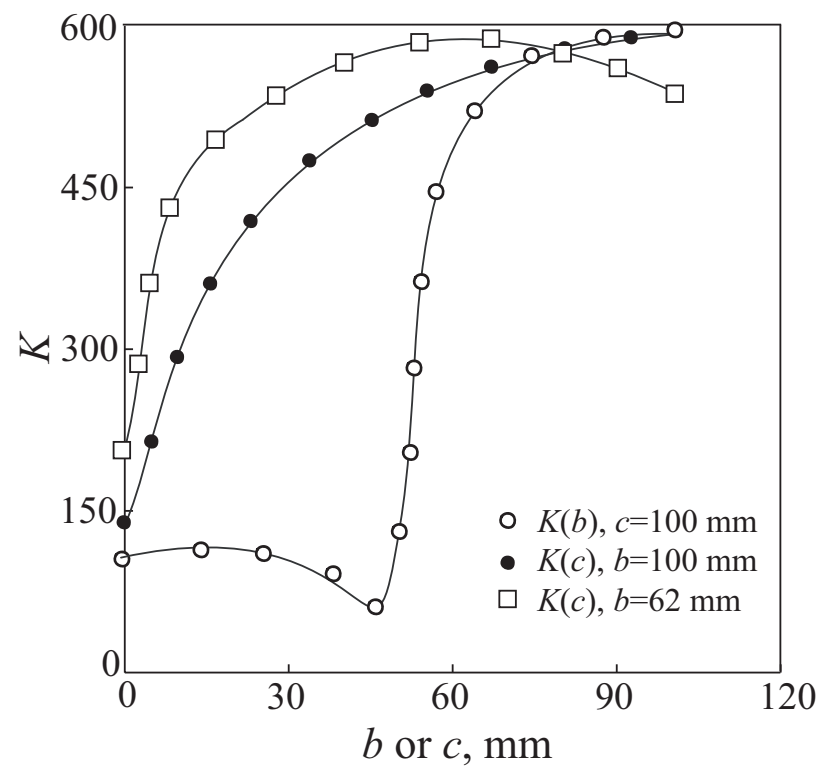

Figure 13. Dependence of the field amplification coefficient at the vibrator edge on the screen dimensions

To find the breakdown pressure, experiments are performed at $b=62 \mathrm{~mm}$ and $H=110$ $\mathrm{mm}$. The breakdown pressure decreases from 615 torr at $c=100 \mathrm{~mm}$ to 480 torr at $c=10$ $\mathrm{mm}$. Two additional measured points correspond to $c=40 \mathrm{~mm}$ and $c=20 \mathrm{~mm}$ when the breakdown pressure remains about the same and equals 600 torr. 


\section{Conclusion}

The discharge is ignited in the presence of an initiator by a quasi-optical beam of microwave radiation with a wavelength of $12.5 \mathrm{~cm}$. A resonant method of initiating a discharge is used, which is more economical than the known plasma ignition and combustion stabilization methods. Streamer discharge absorbs almost all the electromagnetic energy incident on it. Streamers grow into a region of the electromagnetic beam where the amplitude of the electric component of the initial field is lower than the critical breakdown amplitude. For sub-critical streamer discharge, breakdown conditions are created by special initiators within its local region, and the discharge propagates toward the source of electromagnetic beam. Experimental dependencies of the critical field strength on vibrator length are presented.

A resonance electromagnetic vibrator placed on a surface that faces a microwave beam initiates air breakdown in a field the sub-criticality of which is more than when the vibrator is placed in a travelling electromagnetic wave. A deep subcritical discharge attached to an initiator located on a dielectric screen is not blown away by a high-speed flow and confidently ignites the mixture. A discharge ignited on a resonator near a dielectric or metal screen is not blown away by a flow with a velocity ranging from $50 \mathrm{~m} / \mathrm{s}$ to $500 \mathrm{~m} / \mathrm{s}$.

The results obtained could be potentially useful in aerospace applications to ignite air/fuel flow in gas turbine engines and flammable mixtures in hypersonic flying vehicles, combustion chambers of micro-turbines, internal combustion engines.

\section{Acknowledgements}

This work was financially supported by the Ministry of Science and Higher Education of Russian Federation during the implementation of the project "Creating a leading scientific and technical reserve in the development of advanced technologies for small gas turbine, rocket and combined engines of ultra-light launch vehicles, small spacecraft and unmanned aerial vehicles that provide priority positions for Russian companies in emerging global markets of the future", No. FZWF-2020-0015.

\section{References}

[1] Aleksandrov K.V., Grachev L.P., Esakov I.I., Fedorov V.V., Khodataev K.V. Domains of existence of various types of microwave discharge in quasi-optical electromagnetic beams. Technical Physics, 2006, 51(11), pp. 1448-1456.

[2] Smirnov N.N., Betelin V.B., Nikitin V.F., Phylippov Yu.G., Jaye Koo. Detonation engine fed by acetylene-oxygen mixture. Acta Astronautica, 2014, 104(1), pp. 134-146.

[3] Lebedev Yu.A. Microwave discharges at low pressures and peculiarities of the processes in strongly non-uniform plasma. Plasma Sources Science and Technology, 2015, 24(5), 053001.

[4] Ju Y., Sun W. Plasma assisted combustion: dynamics and chemistry. Progress in Energy and Combustion Science, 2015, 48, pp. 21-83.

[5] Adamovich I.V., Choi I., Jiang N., Kim J.-H., Keshav S., Lempert W.R., Mintusov E., Nishihara M., Samimy M., Uddi M. Plasma-assisted ignition and high-speed flow control: non-thermal and thermal effects. Plasma Sources Science and Technology, 2009, 18(3), pp. 034 018-1-034 018-13. 
[6] Knight D., Kolesnichenko Y., Brovkin V., Khmara D., Lashkov V., Mashek I. Interaction of microwave-generated plasma with a hemisphere cylinder at Mach 2.1. AIAA Journal, 2009, 47(12), pp. 2996-3010.

[7] Lashkov V.A., Karpenko A.G., Khoronzhuk R.S., Mashek I.Ch. Effect of Mach number on the efficiency of microwave energy deposition in supersonic flow. Physics of Plasmas, 2016, 23(5), 052305.

[8] Klimov A., Bityurin V., Brovkin V., Vystavkin N., Kuznetsov A., Van Wie D. Optimization of plasma-assisted combustion. AIAA Paper, 2002, 2002-2250.

[9] Klimov A., Bityurin V., Kuznetsov A., Tolkunov B., Vystavkin N., Vasiliev M. External and internal plasma-assisted combustion. AIAA Paper 2004, 2004-1014.

[10] Matveev I., Serbin S. Investigation of a reverse-vortex plasma assisted combustion system. Proceedings of the ASME Heat Transfer Summer Conference, 812 July 2012, Puerto Rico, USA. New York: ASME, 2012. HT2012-58037, 133-140.

[11] Dresvin S., Zverev S., Ivanov D., Matveev I. High frequency induction plasma torches / Plasma Assisted Combustion, Gasification, and Pollution Control. Volume I. Methods of Plasma Generation for PAC. Outskirts Press, 2013, 373462.

[12] Bulat M.P., Bulat P.V., Denissenko P.V., Esakov I.I., Grachev L.P., Volkov K.N., Volobuev I.A. Ignition of lean and stoichiometric air-propane mixture with a subcritical microwave streamer discharge. Acta Astronautica, 2018, 150, pp. 153-161.

[13] Denissenko P.V., Bulat M.P., Esakov I.I., Grachev L.P., Volkov K.N., Volobuev I.A., Upyrev V.V., Bulat P.V. Ignition of premixed air/fuel mixtures by microwave streamer discharge. Combustion and Flame, 2019, 202, 417-422.

[14] Pilla G., Lacoste D.A., Veynante D., Laux C.O. Stabilization of a swirled propaneair flame using nanosecond repetitively pulsed plasma. IEEE Transactions on Plasma Science, 2008, 36(4), pp. 940-941.

[15] Shibkov V.M., Aleksandrov A.A., Chernikov V.A., Ershov A.P., Shibkova L.V. Microwave and direct-current discharges in high-speed flow: fundamentals and applications to ignition. Journal of Propulsion and Power, 2009, 25(1), pp. 123-137.

[16] Pertl F.A., Smith J.E. Electromagnetic design of a novel microwave internal combustion engine ignition source, the quarter wave coaxial cavity igniter. Proceedings of the Institution of Mechanical Engineers. Part D: Journal of Automobile Engineering, 2009, 223(11), pp. 1405-1417.

[17] Bulat M.P., Bulat P.V., Denissenko P.V., Esakov I.I., Grachev L.P., Volkov K.N., Volobuev I.A. Ignition and combustion of air/fuel mixture in a long tube induced by microwave subcritical streamer discharge. Acta Astronautica, 2019, 163, pp. 33-43.

[18] Vedenin P.V., Popov N.A. Propagation of a high-pressure microwave discharge in prebreakdown fields: formation of plasma structures. Journal of Experimental and Theoretical Physics, 2003, 96, pp. 40-52.

[19] Kolesnichenko Yu.F., Brovkin V.G., Khmara D.V., Lashkov V.A., Mashek I.Ch., Ryvkin M.I. Fine structure of MW discharge: evolution scenario. AIAA Paper, 2003-362.

[20] Boeuf J.-P., Chaudhury B., Zhu G. Physics Review Letters, 2010, 104, 015002.

[21] Semenov V.E., Rakova E.I., Glyavin M.Y., Nusinovich G.S. Breakdown simulations in a focused microwave beam within the simplified model. Physics of Plasmas, 2016, 23, 073109 . 
[22] Saifutdinov A.I., Kustova E.V., Karpenko A.G., Lashkov V.A. Dynamics of focused pulsed microwave discharge in air. Plasma Physics Reports, 2019, 45(6), pp. 602-609.

[23] Grachev L.P., Esakov I.I., Khodataev K.V. Microwave streamer discharge in a supersonic air flow. Technical Physics, 1999, 44(11), pp. 1271-1275.

[24] Khodataev K.V. Numerical modeling of the combustion, assisted by the microwave undercritical discharge in supersonic flow. AIAA Paper, 2005, 2005-0985.

[25] Esakov I.I., Grachev L.P., Khodataev K.V., Van Wie D.M. Investigation of the possibility of the application of the undercritical microwave streamer gas discharge for the ignition of a fuel in the jet engine. AIAA Paper, 2001-2939.

[26] Esakov I.I., Grachev L.P., Khodataev K.V., Van Wie D.M. Experiments on propane ignition in high-speed airflow using a deeply undercritical microwave discharge. AIAA Paper, 2004-0840.

[27] Esakov I.I., Grachev L.P., Bychkov V.L., Van Wie D. Investigation of undercritical MW discharge with volumetrically developed streamer structure in propane-air supersonic stream. AIAA Paper, 2006-0790.

[28] Breden D., Raja L.L. Gas heating effects in a nanosecond-pulse streamer discharge interacting with a supersonic $\mathrm{O}_{2}-\mathrm{H}_{2}$ flow. IEEE Transactions on Plasma Science, 2011, 39(11), pp. 2250-2251.

[29] Do H., Im S., Cappelli M.A., Mungal M.G. Plasma assisted flame ignition of supersonic flows over a flat wall. Combustion and Flame, 2010, 157(12), pp. 2298-2305.

[30] Mahadevan S., Raja L.L. Simulations of direct-current air surface plasma discharges in supersonic flow. AIAA Paper, 2009, 2009-1192, pp. 1-14.

[31] Duarte M., Bonaventura Z., Massot M., Bourdon A., Descombes S., Dumont T. A new numerical strategy with space-time adaptivity and error control for multi-scale streamer discharge simulations. Journal of Computational Physics, 2012, 231(3), 1002-1019.

[32] Vinogradov V.A., Komratov D.V., Chirkov A.Yu. Study of propane-air mixture combustion initiation by the formation of microwave plasma in a flow. Journal of Physics: Conference Series, 2018, 1094, 012027 (5 pages). 